perhaps the most important, because it deals with carotene synthesis. And, finally, chapter 10 presents data on the possible value of the species in nutrition and therapy.

Carotene (from Dunaliella) is an apparently harmless yellow pigment, a and perhaps an anti-cancer agent (for which encouraging evidence seems to be accumulating). But here I should mention another caveat. There is some question as to whether 'natural' $\beta$ carotene (rich in the 9-cis form found in precursor of vitamin $\mathrm{A}$, an anti-oxidant

Dunaliella) is nutritionally superior to the synthetic product (all trans, and sold at a considerably lower price on the world market), because the natural form tends to isomerize.

Despite the shortcomings I have mentioned, the book is a valuable contribution to the phycological and biotechnological literature. It is well printed, lavishly bound and expensive. $\square$

Ralph A. Lewin is in the Scripps Institution of Oceanography, University of California, San Diego, California 92093-0202, USA.

\section{Evolution of good taste}

\section{Tristram D. Wyatt and Martin C. Birch}

Insect Chemical Ecology: An Evolutionany Approach. Edited by Bernard D. Roitberg and Murray B. Isman. Chapman and Hall: 1992. Pp.359. £55, \$75 (hbk); £24.95, \$35 (pbk).

WHILE the songs of crickets and grasshoppers have provided biologists with some of their best tools for investigating central evolutionary questions, the largely chemically mediated world of most insects has hardly been touched. This is a pity. Few other animal groups can match the intensity of study given to insect sex pheromones, and as P. L. Phelan suggests, this offers exceptional opportunities to test important questions about, say, the evolution of reproductive isolation, speciation and the relative contribution of sexual selection. The sensory world of most insects is dominated by smell and taste and is involved in finding mates, oviposition sites, food plants and more. In each of these areas there are increasingly well-studied systems ready to be explored by the evolutionary biologist. Evidence for testing conflicting hypotheses awaits recognition.

Genetic engineers splicing genes for bacterial toxins into crop plants to provide resistance to insect herbivores may also be interested. For, as M. A. Caprio and B. E. Tabashnik note, "the remarkable diversity of [natural] plant defensive compounds is matched only by the ability of insects to overcome them". Will defences derived from Bacillus thuringiensis prove to be different?

Evolutionary biologists are one target audience for the book, but more important perhaps are chemical ecologists themselves. Traditionally, insect chemical ecology has focused on the chemicals involved and the immediate questions of the function of behaviours such as mate location by sex pheromones. Evolutionary questions have too often been ignored. Niko Tinbergen's plea to integrate the "four whys" (function, caus- ation; phylogeny and development) has never been more needed, and is a very welcome theme throughout this book.

Our growing knowledge about insect chemical ecology is largely due to three developments: (1) greatly improved techniques for analysis, which have done for pheromones and other chemicals what tape and sonography did for studies of sound (although producing the 'playback' can still be a problem - insects and plants are often better and more precise chemists than we are); (2) a greater knowledge of the sensory physiology of insects; and (3) a more evolutionary approach.

This book is a timely reminder of how productive an evolutionary perspective can be, providing explanations of otherwise baffling problems. Among the topics covered are the evolution of plant-insect interactions, semiochemicals and insect sociality, pheromones and mating systems, and the sensory physiology of chemical perception.

In the last chapter, J. N. McNeil shows how pure and applied scientists, often mutually distrustful and dismissive, could gain from working together. For example, the behavioural ecologists' understanding of alternative mating strategies can help to explain the results of attempts by applied entomologists to use synthetic sex pheromones to disrupt the mating of moths in crops; ecological insights could make pest control more effective; and evolutionary biologists could exploit the knowledge gained from intensive study of pest species.

Chemical ecologists will find this book an invaluable stimulus, encouraging them to pose questions in a more evolutionary and ultimately a more productive way.

Tristram D. Wyatt is in the Departments of Continuing Education and Zoology, and Martin C. Birch is in the Department of Zoology, University of Oxford, Oxford OX1 3PS, UK.

\section{Stellar theory}

\author{
R. J. Tayler
}

Structure and Evolution of Single and Binary Stars. By C. W. H. de Loore and C. Doom. Kluwer: 1992. Pp. 458. DFL 240, £84, \$142 (hbk); DFL 120, £42, $\$ 69$ (pbk).

OVER the past 20 years, astronomers have come to realize that considerable mass is lost from stars at many phases in their evolution and that many of the most dramatic stellar events involve the interaction of two stars in a close binary system. Most textbooks on stellar evolution are largely concerned with the properties of single, spherical, constantmass stars and are therefore not equipped for a discussion of mass loss and binary evolution. But de Loore and Doom have been personally involved in these fields so it is natural that these aspects feature prominently in their book.

The authors' theoretical discussion of the equations of stellar structure and of the physics of stellar interiors is less rigorous and detailed than that of another recent text, Stellar Structure and Evolution by R. Kippenhahn and A. Weigert (Springer, 1990). The main emphasis of de Loore and Doom is on the results of calculations of the evolution of stars of a wide range of masses. When there is uncertainty about the validity of theoretical models, as in the phenomenon known as convective overshooting, they discuss a variety of calculations that indicate how important the uncertainty is. In addition, they tabulate the results of many calculations of the evolution of both single and binary stars. For binary stars, they have also displayed information about the observed properties of many systems. These features will make the book a useful source.

Unfortunately, the text has not been checked very carefully and there are many typographical errors. The ones I noticed should not trouble readers already familiar with the field but will cause some problems for students. More serious is that there seems to be an error in one of the fundamental equations of stellar structure. The authors' energy equation differs from that in other standard texts by including an explicit term for the rate of change of gravitational energy, whereas another term in the equation, the rate of work done by the pressure, includes the change of gravitational energy implicitly. As a result, the set of equations does not conserve energy in rapid evolutionary phases.

R. J. Tayler is at the Astronomy Centre, University of Sussex, Falmer, Brighton BN1 9QH, UK. 